\title{
Image-Quality Assessment for Several Positron Emitters Using the NEMA NU 4-2008 Standards in the Siemens Inveon Small-Animal PET Scanner
}

Jonathan A. Disselhorst ${ }^{1,2}$, Maarten Brom ${ }^{1}$, Peter Laverman ${ }^{1}$, Cornelius H. Slump ${ }^{1}$, Otto C. Boerman ${ }^{1}$, Wim J.G. Oyen ${ }^{1}$, Martin Gotthardt ${ }^{1}$, and Eric P. Visser ${ }^{1}$

${ }^{I}$ Department of Nuclear Medicine, Radboud University Nijmegen Medical Centre, Nijmegen, The Netherlands; and

${ }^{2}$ Institute of Technical Medicine, University of Twente, Enschede, The Netherlands

The positron emitters ${ }^{18} \mathrm{~F},{ }^{68} \mathrm{Ga},{ }^{124} \mathrm{I}$, and ${ }^{89} \mathrm{Zr}$ are all relevant in small-animal PET. Each of these radionuclides has different positron energies and ranges and a different fraction of single photons emitted. Average positron ranges larger than the intrinsic spatial resolution of the scanner (for ${ }^{124} \mathrm{I}$ and ${ }^{68} \mathrm{Ga}$ ) will deteriorate the effective spatial resolution and activity recovery coefficient $(\mathrm{RC})$ for small lesions or phantom structures. The presence of single photons (for ${ }^{124} \mathrm{I}$ and ${ }^{89} \mathrm{Zr}$ ) could increase image noise and spillover ratios (SORs). Methods: Image noise, expressed as percentage SD in a uniform region (\%SD), RC, and SOR (in air and water) were determined using the NEMA NU 4 smallanimal image-quality phantom filled with $3.7 \mathrm{MBq}$ of total activity of ${ }^{18} \mathrm{~F},{ }^{68} \mathrm{Ga},{ }^{124} \mathrm{I}$, or ${ }^{89} \mathrm{Zr}$. Filtered backprojection (FBP), orderedsubset expectation maximization in 2 dimensions, and maximum a posteriori (MAP) reconstructions were compared. In addition to the NEMA NU 4 image-quality parameters, spatial resolutions were determined using small glass capillaries filled with these radionuclides in a water environment. Results: The \%SD for ${ }^{18} \mathrm{~F},{ }^{68} \mathrm{Ga},{ }^{124} \mathrm{I}$, and ${ }^{89} \mathrm{Zr}$ using $\mathrm{FBP}$ was $6.27,6.40,6.74$, and 5.83 , respectively. The respective RCs were $0.21,0.11,0.12$, and 0.19 for the $1-\mathrm{mm}$-diameter rod and $0.97,0.65,0.64$, and 0.88 for the 5 -mm-diameter rod. SORs in air were $0.01,0.03$, 0.04 , and 0.01 , respectively, and in water $0.02,0.10,0.13$, and 0.02 . Other reconstruction algorithms gave similar differences between the radionuclides. MAP produced the highest RCs. For the glass capillaries using FBP, the full widths at half maximum for ${ }^{18} \mathrm{~F},{ }^{68} \mathrm{Ga},{ }^{124} \mathrm{I}$, and ${ }^{89} \mathrm{Zr}$ were $1.81,2.46,2.38$, and $1.99 \mathrm{~mm}$, respectively. The corresponding full widths at tenth maximum were $3.57,6.52,5.87$, and $4.01 \mathrm{~mm}$. Conclusion: With the intrinsic spatial resolution $(\approx 1.5 \mathrm{~mm})$ of this latestgeneration small-animal PET scanner, the finite positron range has become the limiting factor for the overall spatial resolution and activity recovery in small structures imaged with ${ }^{124} \mathrm{I}$ and ${ }^{68} \mathrm{Ga}$. The presence of single photons had only a limited effect on the image noise. MAP, as compared with the other recon-

Received Jul. 26, 2009; revision accepted Jan. 11, 2010.

For correspondence or reprints contact: Jonathan A. Disselhorst, Department of Nuclear Medicine, Internal Postal Code 444, Radboud University Nijmegen Medical Centre, P.O. Box 9101, 6500 HB Nijmegen, The Netherlands.

E-mail: j.disselhorst@nucmed.umcn.nl

COPYRIGHT () 2010 by the Society of Nuclear Medicine, Inc. struction algorithms, increased $\mathrm{RC}$ and decreased \%SD and SOR.

Key Words: positron emission tomography; small-animal; positron range; image quality; NEMA

J Nucl Med 2010; 51:610-617

DOI: 10.2967/jnumed.109.068858

$\mathbf{P}$ ET with ${ }^{18} \mathrm{~F}$ is widely used in a range of applications, using tracers such as ${ }^{18} \mathrm{~F}-\mathrm{FDG},{ }^{18} \mathrm{~F}$-fluoromisonidazole, ${ }^{18} \mathrm{~F}$-fluoroazomycin arabinoside, and ${ }^{18} \mathrm{~F}$-fluoro-3'-deoxy-3'L-fluorothymidine (1). In some applications, other positronemitting radionuclides are being used in the preclinical setting and have also found their way to clinical PET. The use of positron emitter-labeled monoclonal antibodies that combine the specificity of an antibody with the resolution of PET requires radionuclides with half-lives that match the half-life of antibodies in the circulation $(>48 \mathrm{~h})$. With half-lives of 100 and $78 \mathrm{~h}$, respectively, ${ }^{124} \mathrm{I}$ and ${ }^{89} \mathrm{Zr}$ are potentially suitable for this purpose.

In many situations, a generator-produced radionuclide such as ${ }^{68} \mathrm{Ga}$ is preferable over a cyclotron-produced radionuclide (such as ${ }^{18} \mathrm{~F},{ }^{124} \mathrm{I}$, and ${ }^{89} \mathrm{Zr}$ ), because it can be eluted from a generator on-site and does not require a cyclotron in the vicinity of the PET facility. Peptides conjugated to appropriate chelators such as 1,4,7,10-tetraazacyclododecane1,4,7,10-tetraacetic acid or 1,4,7-triazacyclononane-1,4-7-triacetic acid can be labeled with ${ }^{68} \mathrm{Ga}(2)$.

Some of these radionuclides have disadvantageous properties for PET: for instance, their high positron energy and corresponding large positron range in tissue (e.g., ${ }^{68} \mathrm{Ga}$ and ${ }^{124}$ I) may reduce the spatial resolution of the image. Also, the presence of single $\gamma$-photons (e.g., ${ }^{124} \mathrm{I}$ and ${ }^{89} \mathrm{Zr}$ ) can have detrimental effects. Single $\gamma$-photons with energy above the lower discriminator value of the energy window can lead to coincidence detections without spatial correlation with the 
location of the positron emission, leading to an additional, more or less uniform background concentration. Also, higher noise levels can be expected as a result of a higher rate of multiple detections, which effectively decreases the rate of detected true positron annihilation events. Finally, the single $\gamma$-photons may influence the dead-time and associated corrections of the scanner.

The effects of large positron range and additional singles emissions can be corrected to some extent. For a small object centered in the field of view (FOV), the emitted single $\gamma$-photons produce a uniform distribution of counts $(3,4)$, allowing a simple uniform subtraction to correct for single photons. This is, however, not functional in the present scanner software (Inveon Acquisition Workplace 1.2.2.2 [IAW]; Siemens Medical Solutions). The effects of dead time are corrected for to some degree by the dead-time correction algorithm. The reconstruction software can use scatter correction to reduce the adverse effects of scatter of the annihilation photons on the reconstructed images. Correction for positron range has been described by Bai et al. (5) but is still experimental and not yet available in standard reconstruction software for commercial small-animal PET scanners.

It is relevant to establish the performance of a PET scanner for different radionuclides, not only in terms of spatial resolution and sensitivity but especially with regard to overall image quality. Performance evaluation guidelines for clinical PET scanners have been available for a long time (NEMA NU $2(6-8)$ ), but guidelines for small-animal PET have been introduced only recently (NEMA NU 4 (9)). For the Siemens Inveon small-animal PET scanner, performance characterizations have been established earlier using ${ }^{18} \mathrm{~F}$ or ${ }^{22} \mathrm{Na}$ sources (10-13). The purpose of this study was to compare image-quality parameters of ${ }^{18} \mathrm{~F},{ }^{68} \mathrm{Ga},{ }^{124} \mathrm{I}$, and ${ }^{89} \mathrm{Zr}$ using the NEMA NU 4 image-quality phantom.

\section{MATERIALS AND METHODS}

\section{PET Scanner}

The Siemens Inveon is a high-resolution small-animal PET scanner consisting of 4 rings of 16 lutetium oxyorthosilicate detector blocks. The blocks are composed of $20 \times 20$ crystals, each $1.5 \times 1.5 \times 10 \mathrm{~mm}^{3}$. The detector ring diameter of $16.1 \mathrm{~cm}$ and axial length of $12.7 \mathrm{~cm}$ provide a relatively large maximum acceptance angle of $38.3^{\circ}$ (aspect ratio, 0.79), contributing to the high-peak sensitivity exceeding $10 \%(10,11)$. Light guides couple the detector blocks to photomultiplier tubes. Emission data are acquired in list mode and can be sorted into 2-dimensional (2D) or 3-dimensional (3D) sinograms. To correct for photon attenuation, transmission measurements with a rotating ${ }^{57}$ Co point source can be performed.

The reconstruction algorithms available in IAW are filtered backprojection (FBP), 3D reprojection, 2D and 3D ordered-subset expectation maximization (OSEM2D and OSEM3D, respectively), and maximum a posteriori (MAP) reconstruction (14).

\section{Radionuclides}

The radionuclides selected for this study are ${ }^{18} \mathrm{~F}$ - as it is the most widely used and required for standard image-quality measurements by NEMA NU 4-and the following 3 others (with different properties), which are increasingly used in PET: ${ }^{124} \mathrm{I}$ as a radionuclide with a high positron energy and a high abundance of singles, with an energy within the default 350-650 keV window; ${ }^{68} \mathrm{Ga}$, which also emits high-energy positrons but only an insignificant amount of singles; and ${ }^{89} \mathrm{Zr}$, which does not have high-energy positron emission and for which the specific challenge lies in the abundance of singles. With an energy of $909 \mathrm{keV}$, these photons fall outside the default energy window, apart from a portion of the down-scattered photons with lower energy. Their high yield ( $0.99 \gamma$-photons vs. 0.23 positrons) could contribute to the detector dead time. An overview of the physical properties of the radionuclides is presented in Table $1(15,16)$.

${ }^{18} \mathrm{~F},{ }^{124} \mathrm{I}$, and ${ }^{89} \mathrm{Zr}$ were obtained from BV Cyclotron VU. The respective agents were ${ }^{18} \mathrm{~F}-\mathrm{FDG}$, a $\mathrm{Na}^{124} \mathrm{I}$ solution in $0.1 \mathrm{M}$ $\mathrm{NaOH}$, and a desferrioxamine-conjugated antibody labeled with ${ }^{89} \mathrm{Zr}$ in $\mathrm{NaCl}$-gentisic acid with $0.5 \%$ bovine serum albumin. ${ }^{68} \mathrm{GaCl}_{3}$ was eluted with $0.1 \mathrm{M} \mathrm{HCl}$ from a (TiO-based) ${ }^{68} \mathrm{Ge} /{ }^{68} \mathrm{Ga}$ generator (Cyclotron Co.).

\section{NEMA NU 4 Image-Quality Phantom}

The NEMA NU 4-2008 image-quality phantom is a 50-mmlong, 30-mm-diameter cylinder made of polymethylmethacrylate and consists of different regions to analyze 3 distinct aspects of image quality. A schematic view of the phantom is shown in Figure 1. The first $20 \mathrm{~mm}$ of the phantom body are solid, with 5 fillable rods with diameters of 1,2,3, 4, and $5 \mathrm{~mm}$. These are used to determine the recovery coefficient ( $\mathrm{RC}$ ), defined as the ratio between the measured activity concentration in the rods and the

\begin{tabular}{|c|c|c|c|c|}
\hline Property & ${ }^{18} \mathrm{~F}$ & ${ }^{68} \mathrm{Ga}$ & ${ }^{124} \mid$ & $89 \mathrm{Zr}$ \\
\hline Half-life & $109.8 \mathrm{~min}$ & $67.6 \mathrm{~min}$ & $4.18 d$ & $3.27 \mathrm{~d}$ \\
\hline$\beta^{+}$yield & 0.97 & 0.89 & 0.23 & 0.23 \\
\hline Mean $\beta^{+}$energy $(\mathrm{MeV})$ & 0.25 & 0.83 & 0.83 & 0.40 \\
\hline Mean $\beta^{+}$range in water $(\mathrm{mm})$ & 0.62 & 3.48 & 3.48 & 1.23 \\
\hline Single $\gamma$-yield in range of $350-650 \mathrm{keV}$ & 0.00 & 0.00 & 0.64 & 0.00 \\
\hline Single $\gamma$-yield outside range of $350-650 \mathrm{keV}$ & 0.00 & $0.03(1.08 \mathrm{MeV})$ & $0.10(722.8 \mathrm{keV})$ & $0.99(909.2 \mathrm{keV})$ \\
\hline \multicolumn{5}{|c|}{$0.19(>1.5 \mathrm{MeV})$} \\
\hline
\end{tabular}




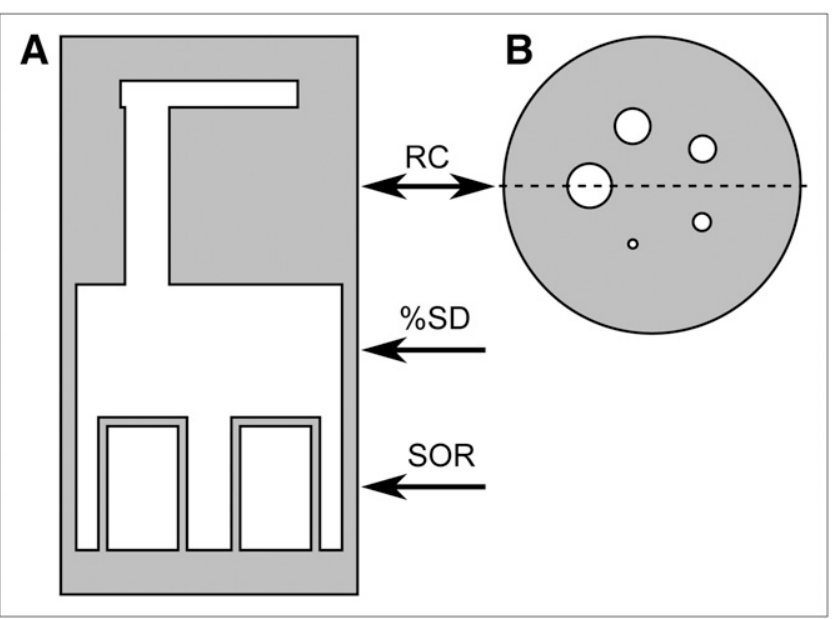

FIGURE 1. Cross-sectional diagram of NEMA NU 4 imagequality phantom. Gray represents solid polymethylmethacrylate, and white represents hollow, fillable compartments. Views are coronal $(A)$ and transverse through rod (B).

activity concentration measured in the uniform area. The RC is theoretically limited between 1 and $0(0<\mathrm{RC} \leq 1)$.

A fillable cylindric chamber with 2 hollow cylinders (length, $14 \mathrm{~mm}$; inner diameter, $8 \mathrm{~mm}$ ) makes up the remaining $30 \mathrm{~mm}$ of the phantom. One of these cylinders is filled with air, the other with nonradioactive water. These 2 cylinders are used to determine the spillover ratio (SOR) in water and air, defined as the mean value in each cold cylinder divided by the mean in the uniform area. Both cylinders are nonradioactive, but as a result of scattered photons, nonzero positron range, randoms, or other effects, the reconstructed images may still display activity in these compartments. The SOR is theoretically limited between 1 and $0(0 \leq \mathrm{RC}<1)$.

The central, uniform region of the phantom is used to determine the percentage SD (\%SD, the SD divided by the mean multiplied by $100 \%)$ as a measure of noise. The phantom was constructed according to the NEMA NU 4 specifications by Agile Engineering.

Before each measurement, the phantom was checked for and cleaned of any activity remaining from previous use. The total volume of the phantom regions filled with activity equaled 20.7 $\mathrm{mL}$. The 5 rods and the large chamber, excluding the cold cylinders, were filled with radioactive solutions with an activity (at the start of the scan) of $3.7 \mathrm{MBq}$ as defined by NEMA.

\section{Glass Capillaries}

In addition to the NEMA NU 4 image-quality assessment, the transaxial spatial resolutions of the 4 radionuclides were determined. According to NEMA specifications, resolution should be measured using ${ }^{22} \mathrm{Na}$ point sources of less than $0.3 \mathrm{~mm}$ in diameter. However, the 4 radionuclides used in this study cannot be easily converted into point sources with these dimensions. Therefore, more practical line sources were used. Glass capillaries of 1.15-mm inner diameter (1.55-mm outer diameter) were filled with radioactive solutions. Activity concentrations of greater than $30 \mathrm{MBq} / \mathrm{mL}$ were used in a volume of about $70 \mu \mathrm{L}$. The glass capillaries were placed in a 2 -cm-diameter plastic tube filled with water.

\section{Data Acquisition and Image Reconstruction}

Settings for NEMA NU 4 Image-Quality Phantom. The standard NEMA NU 4 protocol advises a 20-min emission scan with an initial ${ }^{18} \mathrm{~F}$ activity of $3.7 \mathrm{MBq}$. However, these numbers have been specifically tailored for ${ }^{18} \mathrm{~F}$, and because of the differences in positron yield (or branching fraction) and half-life, the numbers may not be directly applicable for scanning other radionuclides. For a proper comparison of different radionuclides, equal numbers of positrons need to be emitted from the phantom during the scan. This can be achieved by adjusting either the total scan duration or the start activity. In Table 2, the total number of positron emissions that would occur under exact NEMA NU 4 specifications-that is, during a 20-min acquisition with an initial activity of $3.7 \mathrm{MBq}-$ is given in column 1 . The values in column 2 represent the length of acquisition required to obtain $4.03 \times 10^{9}$ positron emissions given a start activity of $3.7 \mathrm{MBq}$. In column 3, the required start activity is given to obtain $4.03 \times 10^{9}$ positron emissions during a 20 -min scan. In this study, the scan duration was adjusted.

The phantom was positioned on the scanner bed and manually centered in the FOV, using the built-in lasers of the scanner for guidance. The phantom always contained $178.7 \mathrm{~Bq} / \mu \mathrm{L}$ (corresponding to a total activity of $3.7 \mathrm{MBq}$ ) at the start of the scan. Default energy and timing window settings of 350-650 keV and $3.432 \mathrm{~ns}$, respectively, were used. Other window settings have not been considered in this study, to allow for comparison with results in the literature and because NEMA NU 4 requires these acquisition parameters to be constant throughout all tests.

For all radionuclides, a transmission scan of the phantom was made for about $3,600 \mathrm{~s}$ with a ${ }^{57} \mathrm{Co}$ point source of $82 \mathrm{MBq}$. Because the scans were not obtained on the same day, the transmission scan durations were adjusted to account for transmission source decay to ensure an identical statistical quality of the attenuation correction.

All default settings were used for histogramming; that is, the emission data were histogrammed into $3 \mathrm{D}$ sinograms with a span of 3 and a maximum ring difference of 79, delays were subtracted, and global dead-time correction was applied. The transmission data were rebinned into single slices, with a span of 17 and a ring difference of 42, and global dead-time correction was applied.

The PET data were reconstructed with various algorithms to compare their performance for different radionuclides. The image matrix size was $256 \times 256 \times 159$ with pixel sizes of $0.43 \times 0.43 \times$ $0.80 \mathrm{~mm}^{3}$ for MAP and $0.39 \times 0.39 \times 0.80 \mathrm{~mm}^{3}$ for FBP and OSEM2D. FBP was used with a ramp filter (cutoff at Nyquist frequency), and OSEM2D was performed using 4 iterations and 16 subsets. Before these 2D reconstructions, the 3D sinogram data

TABLE 2. Required Scan Durations or Start Activities to Obtain Equal Numbers of Emitted Positrons for Each Radionuclide

\begin{tabular}{|c|c|c|c|}
\hline Radionuclide & $\begin{array}{c}\text { Number of positron } \\
\text { emissions }\left(\times 10^{9}\right) \\
\text { using NEMA } \\
\text { specifications }\end{array}$ & $\begin{array}{l}\text { Corrected } \\
\text { scan } \\
\text { duration (s) }\end{array}$ & $\begin{array}{c}\text { Corrected } \\
\text { start activity } \\
(\mathrm{MBq})\end{array}$ \\
\hline${ }^{18} \mathrm{~F}$ & 4.03 & 1,200 & 3.70 \\
\hline${ }^{68} \mathrm{Ga}$ & 3.58 & 1,373 & 4.17 \\
\hline $124 \mid$ & 1.03 & 4,707 & 14.47 \\
\hline${ }^{89} \mathrm{Zr}$ & 1.01 & 4,824 & 14.81 \\
\hline
\end{tabular}


were Fourier rebinned. OSEM3D-MAP reconstructions were performed using 2 OSEM3D iterations and 16 subsets, followed by 18 MAP iterations. The smoothing factor $\beta$ in MAP reconstructions was set to 0.1 , and the uniformity constraint was set to uniform variance. These settings led to the most favorable results using ${ }^{18} \mathrm{~F}$, considering both $\mathrm{RC}$ and \%SD (unpublished results).

All MAP reconstructions were preceded by 2 OSEM3D iterations, because OSEM3D converges faster than MAP. Here, MAP will refer to this combined OSEM3D and MAP reconstruction.

IAW provides a scatter-correction algorithm based on direct calculation from analytic formulas and source and object geometry (17). Although NEMA recommends reconstruction with all corrections applied, we opted for reconstruction with and without scatter correction, mainly because of some unexpected deformations induced by the correction algorithm.

Settings for Glass Capillaries. The glass capillaries were positioned in the center of the FOV, aligned with the axis of the scanner, and scanned until at least 3.5 million counts were acquired using the default energy window of 350-650 keV and timing window of $3.432 \mathrm{~ns}$. Images were obtained using FBP with the default settings (ramp filter with a cutoff at Nyquist frequency) and $512 \times 512 \times 159$ matrices with pixel sizes of $0.19 \times 0.19 \times$ $0.80 \mathrm{~mm}$. This matrix size was selected to obtain a profile with a sufficient number of datapoints to allow for the accurate determination of spatial resolution. Attenuation and scatter corrections were not applied.

\section{Image Analysis}

The reconstructed images were processed with a program written in Matlab (version R2008a; The MathWorks) to determine the various image-quality parameters and full width at half and at tenth maximum (FWHM and FWTM, respectively) for the glass capillaries. The program provided accurate and reproducible placement of volumes of interest (VOIs) in all images.

The $\%$ SD were determined in a 22.5 -mm-diameter $(75 \%$ of the inner phantom diameter), 10-mm-long cylindric VOI drawn over the center of the uniform region of the phantom.

To determine the RCs, the image slices containing the central $10 \mathrm{~mm}$ of the rods were averaged and circular regions of interest were drawn around each rod with a diameter twice their physical diameter. The positions of the maximum values in these regions of interest were used to create line profiles in the axial direction through the rods. The RCs were determined from the mean values along these 5 profiles, divided by the mean activity concentration in the uniform area.

Two 4-mm-diameter (50\% of the physical inner diameter), 7.5mm-long cylindric VOIs were drawn over the center of the air- and water-filled compartments. The means of these cold regions divided by the mean of the uniform radioactive area provide the SOR.

As mentioned above, the NEMA NU 4 image-quality guidelines have been created for the use of ${ }^{18} \mathrm{~F}$, but with a few adjustments these can also be used for other radionuclides. However, the determination of the SORs is not completely applicable to long-range positron emitters. Because the radius of the scatter compartments is relatively small, the measured activity in these regions not only is due to scattered photons but also contains contributions from positrons emitted in the body part of the phantom and annihilating in the scatter compartments. For a fair comparison, the 2 effects should be separated. Decreasing the diameter of the VOI in the scatter compartments is the only method achievable in the NEMA NU 4 image-quality phantom, but the diameter of the compartments is too small to fully eliminate positron range effects. However, by assessing the difference in SOR between the compartments filled with water and air, the accuracy of corrections can be evaluated. Whereas the SOR in water comprises photon scatter and the effect of positron range, only scattered photons contribute to the SOR in air; because of the large positron range $(>1 \mathrm{~m})$, almost no annihilations occur in air.

\section{Capillary Measurements}

The slices containing the capillary in the reconstructed images were aligned by placing their maximum in the center before being summed to form a single image. Profiles in tangential and radial directions through the center were averaged to determine the FWHM and FWTM for all radionuclides. These widths were determined according to NEMA NU 4 guidelines: maxima were determined in a parabolic fit through the peak pixel and its 2 nearest neighboring pixels. The FWHM and FWTM were determined by linear interpolation between adjacent pixels at half and one tenth of this maximum, respectively. FWHM and FWTM were not corrected for source geometry.

\section{RESULTS}

In the following sections, the results for each of the NEMA NU 4 image-quality parameters are presented. A complete list of all the obtained parameter values can be found in Supplemental Tables 1-4 (supplemental materials are available online only at http://jnm.snmjournals.org).

\section{Spatial Resolution}

Results of the spatial resolution measurements using the glass capillaries are summarized in Table 3 . For gaussian profiles, the FWHM-to-FWTM ratio equals $\sqrt{\ln 2 / \ln 10} \approx$

\section{TABLE 3. Spatial Resolutions for 4 Radionuclides Measured with Glass Capillaries Surrounded by Water}

\begin{tabular}{|c|c|c|c|c|c|}
\hline \multirow[b]{2}{*}{ Radionuclide } & \multirow{2}{*}{$\begin{array}{c}\text { Mean } \beta+ \\
\text { energy }(\mathrm{MeV})\end{array}$} & \multicolumn{3}{|c|}{ Measured profiles } & \multirow{2}{*}{$\begin{array}{c}\text { Palmer et al. (18), } \\
\text { FWHM-to-FWTM (mm) }\end{array}$} \\
\hline & & FWHM (mm) & FWTM (mm) & FWHM-to-FWTM (mm) & \\
\hline${ }^{18} \mathrm{~F}$ & 0.25 & 1.81 & 3.57 & 0.51 & 0.54 \\
\hline${ }^{68} \mathrm{Ga}$ & 0.83 & 2.46 & 6.52 & 0.38 & 0.39 \\
\hline${ }^{124} \mid$ & 0.83 & 2.38 & 5.87 & 0.41 & 0.39 \\
\hline $89 \mathrm{Zr}$ & 0.40 & 1.99 & 4.01 & 0.50 & 0.50 \\
\hline
\end{tabular}


0.55. Nongaussian profiles with extended tails are characterized by smaller FWHM-to-FWTM ratios. Palmer et al. (18) have modeled spatial resolutions for a hypothetical imaging system with an intrinsic spatial resolution of $1.5 \mathrm{~mm}$. Corresponding FWHM-to-FWTM ratios have been added for comparison, as well as the mean positron energies. The data in Table 3 indicate that ${ }^{18} \mathrm{~F}$ yielded the best approximation (0.51) of the theoretic gaussian ratio. The measured and modeled ratios for ${ }^{68} \mathrm{Ga}(0.38$ and 0.39 , respectively) and ${ }^{124} \mathrm{I}$ ( 0.41 and 0.39 , respectively) are substantially lower, indicating a larger tail section of the curve. Figure 2 shows the measured profiles.

\section{Uniformity}

The $\%$ SD of the NEMA NU 4 image-quality phantom is shown in Figure 3. The largest differences are not found among different radionuclides but among the various reconstruction algorithms for the same radionuclide. MAP yields more than 2 times smaller \%SD values than OSEM2D and FBP. The differences per reconstruction algorithm between the 4 radionuclides are much smaller, with a maximum SD of 0.8 percentage point for MAP. Scatter correction had a limited effect on $\% \mathrm{SD}$, with a maximum decrease of 0.8 percentage point for ${ }^{124} \mathrm{I}$ in the OSEM2D images.

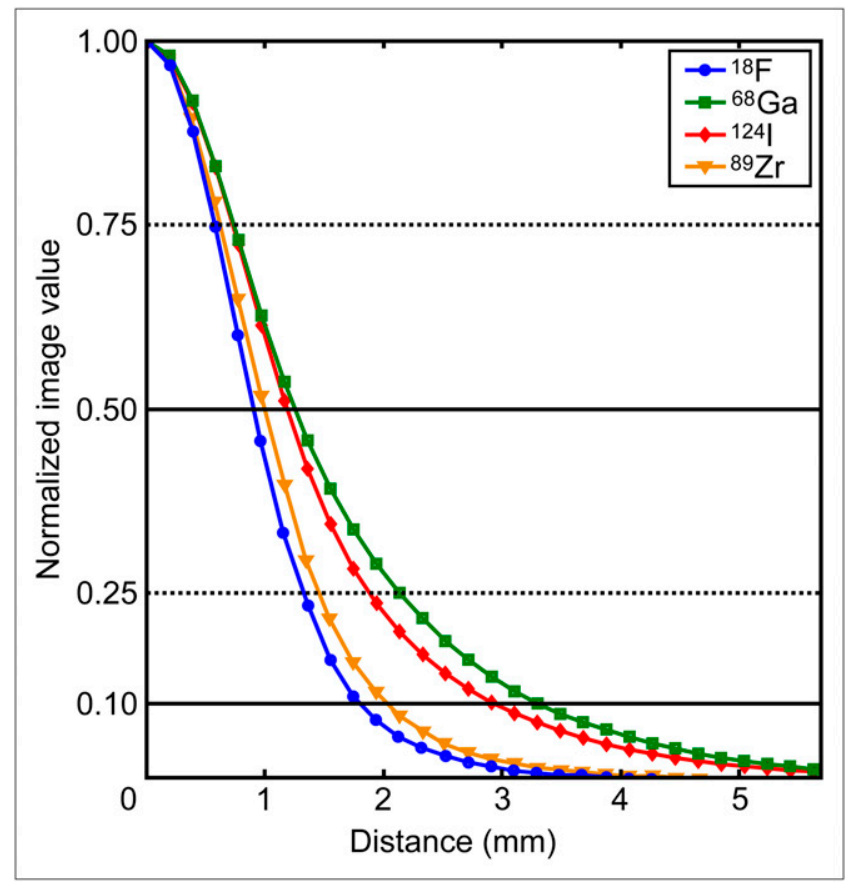

FIGURE 2. Measured line profiles through glass capillaries filled with positron-emitting aqueous solutions, surrounded by water. Profiles were obtained from $512 \times 512$ FBP reconstruction by aligning and summing image slices containing capillary and averaging profiles in tangential and radial directions through center. Profiles are shown in 1 direction only. Values were not corrected for source dimensions.



FIGURE 3. \%SD in uniform phantom region. *Reconstruction performed with scatter correction.

$\mathrm{RCs}$

The RCs of the 5 different rods are shown in Figure 4. Because the result obtained with ${ }^{89} \mathrm{Zr}$ is similar to that obtained with ${ }^{18} \mathrm{~F}$, and ${ }^{124} \mathrm{I}$ is similar to ${ }^{68} \mathrm{Ga}$, the 4 radionuclides can be clearly separated into 2 groups: the long- and short-range positron emitters. The influence of scatter correction on RC was limited (data not shown). The largest differences in RC were introduced by the choice of reconstruction algorithm. This especially holds for the short-range positron emitters, for which the 2D reconstruction algorithms clearly showed lower RC values than did reconstruction with MAP, with a maximum difference between OSEM2D and MAP of 36\%. With MAP, for large-diameter rods, the recovery even rose beyond the ideal limit of 1 . This effect could possibly be attributed to an overshoot at the edges, commonly referred to as the Gibbs effect (19).

For the long-range positron emitters, the differences in RC were smaller, but FBP and OSEM2D still showed lower values than did MAP, with a maximum difference between OSEM2D and MAP of $18 \%$.

\section{Accuracy of Corrections}

In Figure 5, the SORs in the water and air compartments are shown for the 4 radionuclides for different reconstruction algorithms with and without scatter correction. The differences in SOR were large, especially in water. Again, 2 groups can be distinguished: the short-range positron 


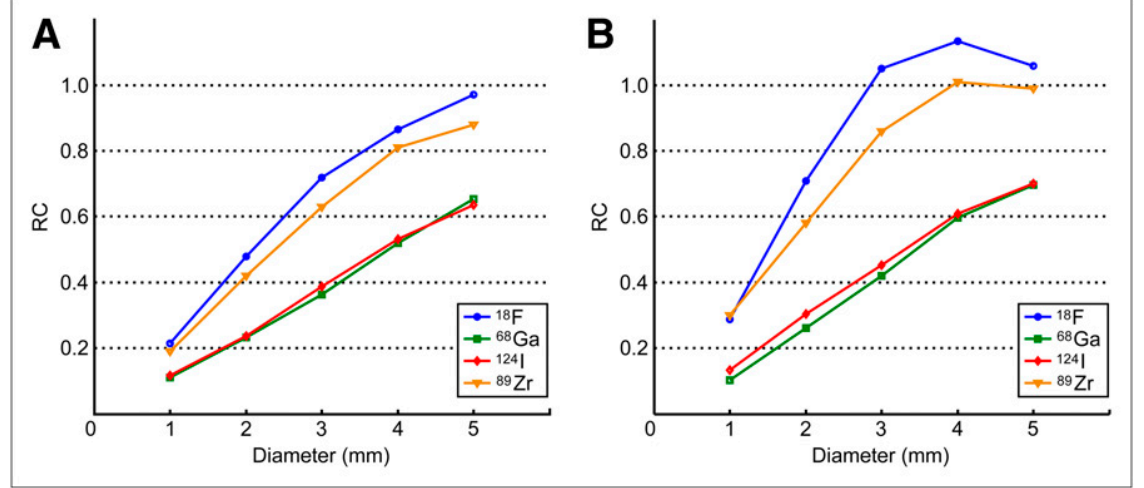

FIGURE 4. RCs of different rods for various radionuclides, reconstructed with FBP (A) and MAP (B). OSEM2D results were similar to those of FBP. emitters, with a clearly lower SOR (mean value for all reconstruction algorithms, 0.03 for ${ }^{18} \mathrm{~F}$ and 0.04 for ${ }^{89} \mathrm{Zr}$ ), and the large-range positron emitters (mean value for all reconstruction algorithms, 0.10 for ${ }^{68} \mathrm{Ga}$ and 0.14 for ${ }^{124} \mathrm{I}$ ). A reduction in SOR was achieved by MAP, as compared with OSEM2D and FBP. In the case of air, this reduction is evident for all radionuclides except ${ }^{89} \mathrm{Zr}$. In the case of water, the reduction is obvious only for ${ }^{18} \mathrm{~F}$. Scatter correction lowered SOR, especially for ${ }^{124} \mathrm{I}$.

Two unexpected features were also observed in the data. First, MAP performed poorly without scatter correction in the case of ${ }^{124} \mathrm{I}$. The SOR was lowered substantially (a decrease of 0.09 ) with scatter correction enabled, possibly related to the abundance of single $\gamma$-photons in the 350$650 \mathrm{keV}$ energy window. Second, only for ${ }^{89} \mathrm{Zr}$, MAP produced a relatively high SOR in air $(0.05)$, which is even higher than the SOR in water (0.04).

The scatter-correction algorithm included in IAW introduced certain deformation artifacts in some parts of the reconstructed images, as shown in Figure 6. The ${ }^{124}$ I image, especially, was affected, whereas the effect was not prominent in the images of the other radionuclides. This could again be related to an abundance of single $\gamma$-photons for ${ }^{124} \mathrm{I}$ in the $350-650 \mathrm{keV}$ energy window, for which correction was unavailable. No abnormalities, however, arose in the NEMA NU 4 parameters by this artifact.

\section{DISCUSSION}

From the results for the RCs of the small rods and capillary measurements, this study shows that the positron range limits the spatial resolution for modern small-animal PET scanners. This is in line with the observations by Liu and Laforest (20), who analyzed 5 different radionuclides $\left({ }^{18} \mathrm{~F},{ }^{61} \mathrm{Cu},{ }^{68} \mathrm{Ga},{ }^{94 \mathrm{~m}} \mathrm{Tc}\right.$, and $\left.{ }^{86} \mathrm{Y}\right)$. These were, however, not measured using the standard NEMA NU 4 image-quality phantom and parameter definitions and were for a different type of scanner (microPET Focus 120; Siemens).

The SORs in water were considerably higher for ${ }^{124} \mathrm{I}$ and ${ }^{68} \mathrm{Ga}$ than for ${ }^{18} \mathrm{~F}$ and ${ }^{89} \mathrm{Zr}$. These results should, however, be interpreted carefully. The high SOR values for the longrange positron emitters in water do not imply incorrect scatter- or other correction algorithms for these radionuclides, because they are largely caused by positrons emitted in the body part of the phantom and annihilating in the water-filled scatter compartment. Selecting a smaller VOI within the scatter compartments could partly improve this. However, with a maximum positron range of more than 10
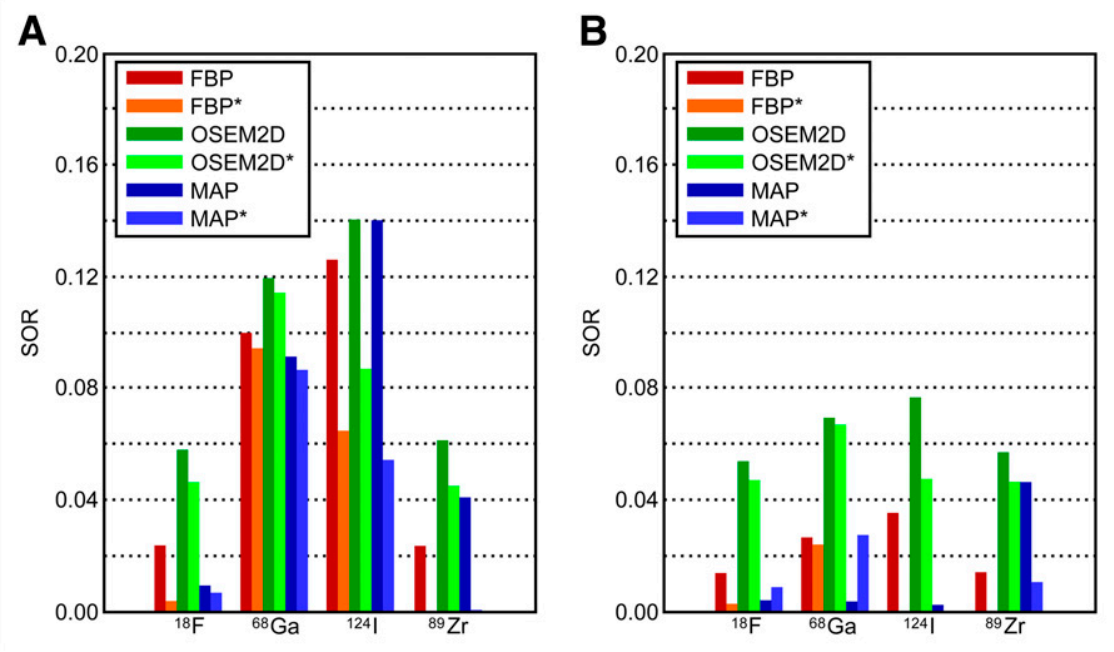

FIGURE 5. SORs in water (A) and air (B) compartments for various radionuclides, reconstructed with 3 different algorithms. Some SOR values are (close to) zero, and not visible in graph. ${ }^{*}$ Reconstruction performed with scatter correction. 

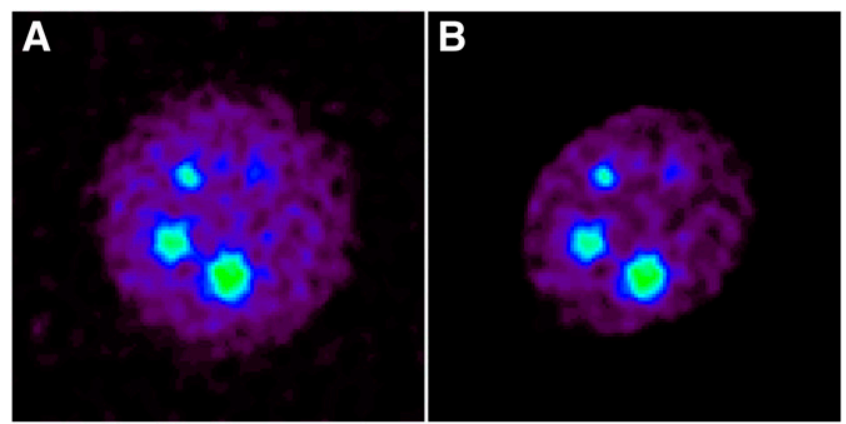

FIGURE 6. Transverse cross-section through rod area of phantom filled with ${ }^{124}$ I solution. Images without $(A)$ and with (B) scatter correction are shown. Scatter-corrected image clearly displays ellipsoid deformation.

$\mathrm{mm}$ and an inner diameter of the compartment of only 8 $\mathrm{mm}$, no valid volume can be drawn. For a real evaluation of the SOR in water, either a different phantom should be used, or correction for positron range should be applied during reconstruction. In air, the average positron range for all radionuclides is greater than $1 \mathrm{~m}$, and scattered and single photons will be the main contributors to the SOR in air, allowing analysis of correction algorithm performance.

As mentioned before, the additional single $\gamma$-photons for ${ }^{124} \mathrm{I}$ and ${ }^{89} \mathrm{Zr}$ could have contributed to the image noise, resulting in a higher \%SD. However, this was not observed. Activities normally injected in small animals $(<10 \mathrm{MBq})$ remain well below the maximum noise-equivalent counting rate measured by Bao et al. (10) and Kemp et al. (12) for ${ }^{18} \mathrm{~F}$. System dead time does not play a large role for these activities. As the activity in the FOV increases, increasing numbers of multiple detections will occur. No differences (in count-rate performance) are to be expected for ${ }^{68} \mathrm{Ga}$, ${ }^{124} \mathrm{I}$, and ${ }^{89} \mathrm{Zr}$, because the total number of emitted $\gamma$-photons (both annihilation and single $\gamma$-photons) is lower for these 3 radionuclides than for ${ }^{18} \mathrm{~F}$.

In addition to image quality, accurate quantification is an important factor in PET. There are 2 issues concerning quantification, one of which depends on a properly performed cross-calibration. During this procedure, a known amount of radioactivity is used in a calibration phantom. With a scaling factor, the measured PET counts per pixel are correlated to the true activity concentration. Another quantification issue concerns the proper scaling of regions with different activity concentrations within the same phantom or animal. In the case of large numbers of single photons (e.g., for ${ }^{124} \mathrm{I}$ ), this could be a problem. Without correction, these singles give an additive, nearly uniform contribution to the activity concentrations in all regions. However, a fixed scaling factor obtained from crosscalibration will lead to concentration-dependent quantification errors, because the spurious activity concentration from the single emissions should have been subtracted before applying the scaling. Because the NEMA NU 4 image-quality phantom contains 1 activity concentration (apart, of course, from all cold regions with zero activity), performance measurements with respect to quantification should be the subject of further study.

In earlier studies with PET radionuclides that emitted single $\gamma$-photons in the acquisition energy window-such as ${ }^{124} \mathrm{I}(21-23),{ }^{76} \mathrm{Br}(3,24-26)$, and ${ }^{86} \mathrm{Y}(24,27-29)$-quantification proved to be affected by the single $\gamma$-photons. Both under- and overestimation of activity concentration occurred, depending on the activity concentration used for cross-calibration.

Basically, MAP performs better than the other 2 algorithms for all 3 image-quality parameters. Accurate modeling of the system response and the statistical nature of the data and incorporation of these during reconstruction improves the resolution recovery of the MAP algorithm. Another distinctive feature of the MAP algorithm is the smoothing factor $\beta$, which regulates the variance and resolution in the image (14). Both aspects improve the image quality of MAP, as compared with both FBP and OSEM2D.

\section{CONCLUSION}

In terms of image-quality parameters, the Inveon smallanimal PET scanner performs best for ${ }^{18} \mathrm{~F}$. The largest differences in the RC and SOR are found between the group of radionuclides with large positron ranges $\left({ }^{124} \mathrm{I}\right.$ and $\left.{ }^{68} \mathrm{Ga}\right)$ and the one with short ranges $\left({ }^{18} \mathrm{~F}\right.$ and $\left.{ }^{89} \mathrm{Zr}\right)$. Only the $\% \mathrm{SD}$ is unaffected by the positron range.

The abundance of single $\gamma$-photons affects only slightly the image-quality parameters; all 4 radionuclides yield roughly the same values for $\% \mathrm{SD}$.

Although scatter correction leads in some cases to deformation artifacts in the images, this technique is beneficial for the SOR and affects only slightly the \%SD and RC. Especially with respect to the RC and \%SD, the MAP algorithm performs much better than do the other reconstruction techniques.

\section{ACKNOWLEDGMENT}

This work was supported by NIH grant 1R01 AG 030328-01.

\section{REFERENCES}

1. Groves AM, Win T, Haim SB, Ell PJ. Non- $\left[{ }^{18} \mathrm{~F}\right]$ FDG PET in clinical oncology. Lancet Oncol. 2007;8:822-830.

2. Tanaka K, Fukase K. PET imaging of biomolecules using metal-DOTA complexes: a new collaborative challenge by chemists, biologists, and physicians for future diagnostics and exploration of in vivo dynamics. Org Biomol Chem. 2008;6:815-828

3. Laforest R, Liu X. Cascade removal and microPET imaging with ${ }^{76} \mathrm{Br}$. Phys Med Biol. 2009;54:1503-1531.

4. Bigott HM, Laforest R, Liu X, et al. Advances in the production, processing and microPET image quality of technetium-94m. Nucl Med Biol. 2006;33:923-933.

5. Bai B, Ruangma A, Laforest R, Tai Y, Leahy RM. Positron range modeling for statistical PET image reconstruction. IEEE Nucl Sci Symp Conf Rec. 2003; $4: 2501-2505$. 
6. National Electrical Manufacturers Association (NEMA). NEMA Standards Publication NU 2-1994: Performance Measurement of Positron Emission Tomographs. Washington, DC: NEMA; 1994.

7. National Electrical Manufacturers Association (NEMA). NEMA Standards Publication NU 2-2001: Performance Measurement of Positron Emission Tomographs. Washington, DC: NEMA; 2001.

8. National Electrical Manufacturers Association (NEMA). NEMA Standards Publication NU 2-2007: Performance Measurement of Positron Emission Tomographs. Washington, DC: NEMA; 2007.

9. National Electrical Manufacturers Association (NEMA). NEMA Standards Publication NU 4-2008: Performance Measurement for Small Animal Positron Emission Tomographs. Washington, DC: NEMA; 2008.

10. Bao Q, Newport D, Chen M, Stout DB, Chatziioannou AF. Performance evaluation of the Inveon dedicated PET preclinical tomograph based on the NEMA NU-4 standards. J Nucl Med. 2009;50:401-408.

11. Visser EP, Disselhorst JA, Brom M, et al. Spatial resolution and sensitivity of the Inveon small-animal PET scanner. J Nucl Med. 2009;50:139-147.

12. Kemp BJ, Hruska CB, McFarland AR, Lenox MW, Lowe VJ. NEMA NU 2-2007 performance measurements of the Siemens Inveon preclinical small animal PET system. Phys Med Biol. 2009;54:2359-2376.

13. Constantinescu CC, Mukherjee J. Performance evaluation of an Inveon PET preclinical scanner. Phys Med Biol. 2009;54:2885-2899.

14. Qi J, Leahy RM, Cherry SR, Chatziioannou AF, Farquhar TH. High-resolution 3D Bayesian image reconstruction using the microPET small-animal scanner. Phys Med Biol. 1998;43:1001-1013.

15. Health Physics Society (HPS). Radionuclide Decay Data. Available at: http:// hps.org/publicinformation/radardecaydata.cfm. Accessed February 16, 2010.

16. International Commission on Radiation Units and Measurements (ICRU). Stopping Powers for Electrons and Positrons. ICRU report 37. Bethesda, MD: ICRU; 1984.

17. Watson CC. New, faster, image-based scatter correction for 3D PET. IEEE Nucl Sci Symp Conf Rec. 1999;3:1637-1641.
18. Palmer MR, Zhu X, Parker JA. Modeling and simulation of positron range effects for high resolution PET imaging. IEEE Trans Nucl Sci. 2005;52:1391-1395.

19. Snyder DL, Miller MI, Thomas LJ, Politte DG. Noise and edge artifacts in maximum-likelihood reconstructions for emission tomography. IEEE Trans Med Imaging. 1987;6:228-238.

20. Liu X, Laforest R. Quantitative small animal PET imaging with nonconventional nuclides. Nucl Med Biol. 2009;36:551-559.

21. Pentlow KS, Graham MC, Lambrecht RM, et al. Quantitative imaging of iodine124 with PET. J Nucl Med. 1996;37:1557-1562.

22. Herzog H, Tellman L, Qaim SM, et al. PET quantitation and imaging of the nonpure positron-emitting iodine isotope ${ }^{124}$ I. Appl Radiat Isot. 2002;56:673-679.

23. Gregory RA, Hooker CA, Partridge M, Flux GD. Optimization and assessment of quantitative ${ }^{124}$ I imaging on a Philips Gemini dual GS PET/CT system. Eur J Nucl Med Mol Imaging. 2009;36:1037-1048.

24. Beattie BJ, Finn RD, Rowland DJ, Pentlow KS. Quantitative imaging of bromine-76 and yttrium-86 with PET: a method for the removal of spurious activity introduced by cascade gamma rays. Med Phys. 2003;30:2410-2423.

25. Ribeiro MJ, Almeida P, Strul D, et al. Comparison of fluorine- 18 and bromine-76 imaging in positron emission tomography. Eur J Nucl Med. 1999;26:758-766.

26. Lubberink M, Schneider H, Bergström M, Lundqvist H. Quantitative imaging and correction for cascade gamma radiation of ${ }^{76} \mathrm{Br}$ with 2D and 3D PET. Phys Med Biol. 2002;47:3519-3534.

27. Walrand S, Jamar F, Mathieu I, et al. Quantitation in PET using isotopes emitting prompt single gammas: application to yttrium-86. Eur J Nucl Med Mol Imaging. 2003;30:354-361.

28. Buchholz HG, Herzog H, Förster GJ, et al. PET imaging with yttrium-86: comparison of phantom measurements acquired with different PET scanners before and after applying background subtraction. Eur J Nucl Med Mol Imaging. 2003;30:716-720.

29. Kull T, Ruckgaber J, Weller R, Reske S, Glatting G. Quantitative imaging of yttrium-86 PET with the ECAT EXACT HR + in 2D mode. Cancer Biother Radiopharm. 2004;19:482-490. 